\title{
Signal-sequence-independent secretion of the staphylococcal nuclease in Mycobacterium smegmatis
}

\author{
Chiara Recchi, Jean Rauzier, Brigitte Gicquel and Jean-Marc Reyrat
}

Author for correspondence: Jean-Marc Reyrat. Tel: +331406132 74. Fax: +3314568 8843. e-mail: jmreyrat@pasteur.fr

Unité de Génétique Mycobactérienne, Institut Pasteur, 25, Rue du Dr Roux, 75724 Paris cedex 15, France

\begin{abstract}
Staphylococcus aureus nuclease is a small, secreted protein which has been successfully used as a reporter system to identify exported products in Lactococcus lactis. Here, biochemical evidence is provided that the nuclease is exported by Mycobacterium smegmatis in the presence, but also in the absence of a signal sequence, and thus probably independently of the Sec translocation pathway. This implies that the nuclease should not be used as a reporter system in mycobacteria for the identification of exported products, despite what has been reported previously in the literature. The nuclease can be extended to create hybrid proteins that remain compatible with its secretion, whereas some other shorter fusions are not tolerated. This suggests that correct folding is required for efficient export. Extensive mutational analysis did not identify a specific secretion pathway. This suggests that the nuclease may be exported by different redundant systems or that components of this alternative Sec pathway are essential for bacterial survival.
\end{abstract}

Keywords: mycobacteria, leaderless secretion

\section{INTRODUCTION}

Much attention has recently been given to the secreted products of Mycobacterium tuberculosis, the causative agent of tuberculosis, a worldwide infectious disease whose virulence has not yet been fully characterized. Exported proteins are thought to have a key role in the disease caused by this slow-growing bacterium by acting either as virulence factors or as antigens modulating the host immune responses.

Biochemical, bioinformatic and genetic approaches have been used to identify M. tuberculosis secreted proteins. Two-dimensional gel electrophoresis and microsequencing have recently identified novel secreted proteins in culture filtrates of M. tuberculosis (Weldingh et al., 1998; Rosenkrands et al., 2000). The recently published M. tuberculosis genome (Cole et al., 1998) and the development of new software have allowed the $M$. tuberculosis genome to be extensively analysed and the identification of proteins which had not been previously predicted to be secreted (Gomez et al., 2000). Conversely, the fusion of genes to reporter proteins whose activities are easily detected on Petri dishes, such as Escherichia coli $\beta$-lactamase (Bla) (Chubb et al., 1998) and alkaline phosphatase (PhoA) (Lim et al., 1995), has identified the coding sequences which trigger the export of the reporter proteins. phoA has been used to create fusion libraries (Lim et al., 1995; Wiker et al., 2000; Carroll et al., 2000) and as a reporter in an in vitro transposition system (Braunstein et al., 2000). In both cases, the methods have proven to be successful, although they are limited to fast-growing mycobacteria.

Recently, a new reporter system based on the Staphylococcus aureus nuclease (Nuc) was designed to identify exported proteins. This small secreted endonuclease has proved to be an ideal reporter system in Lactococcus lactis, in which the truncated gene, deprived of its signal sequence, has been successfully used to screen a fusion library for exported proteins (Poquet et al., 1998) and also to develop an in vitro transposition system (Ravn et al., 2000). Nuc has several advantages over PhoA: it is a stable monomeric protein and its detection assay is nontoxic for cells. This system has recently been adapted for use in mycobacteria and has led to the characterization of three secreted proteins (Downing et al., 1999).

To produce an exhaustive list of M.tuberculosis secreted products we sought to use the nuclease as a reporter system but to detect nuclease activity by a more discriminative method (Lachica et al., 1971). Contrary to our expectations, we found that the staphylococcal nuclease is exported in Mycobacterium smegmatis 
irrespective of whether it was made with or without a signal sequence. Biochemical analysis confirmed that the staphylococcal nuclease is secreted in the absence of bacterial lysis. Although Nuc can be fused to 40 amino acids in the $\mathrm{N}$ terminus without affecting secretion, some other fusions are not compatible with secretion. Despite extensive mutational analysis, we were not able to characterize components of the secretion apparatus which are responsible for this non-canonical export.

\section{METHODS}

Bacterial strains and culture. M. smegmatis $\mathrm{mc}^{2} 155$ and E. coli DH5 $\alpha$ were grown at $37^{\circ} \mathrm{C}$ in L-broth or on L-broth agar, supplemented with kanamycin $\left(20 \mu \mathrm{g} \mathrm{m}^{-1}\right)$ or hygromycin $\left(50 \mu \mathrm{g} \mathrm{ml}^{-1}\right)$ as required. M. smegmatis growth medium was supplemented with $0.05 \%$ Tween 80. Competent E. coli cells were prepared by the $\mathrm{CaCl}_{2}$ method and transformed (Sambrook et al., 1989). Competent M. smegmatis cells were prepared and transformed by electroporation (Guilhot et al., 1992).

Construction of pPRB4 and derivatives. Oligonucleotides are listed in Table 1.

The PCR product of pVE3512 generated by primers A and B was cloned into pPV24 (Triccas et al., 1999) that had been digested with BamHI and KpnI. This gave rise to pPRB4.

$\mathrm{BlaF}^{*}$ derivatives were constructed as follows. pIPJ47* (Timm et al., 1994) PCR products were amplified with primers BlaPf and BlaPr for pBla1, primers BlaPf and BlaSr for pBla2, and primers BlaSf and BlaSr for pBla3. The pIPJ47* PCR products were digested with BamHI and PstI and inserted into the dephosphorylated Bam HI/PstI-digested pPRB4 vector.

SigE derivatives were obtained by cloning the following PCR products into pPRB4. pSigE1 was obtained by amplifying pIPX70 with primers SigEPf and SigErev, and pSigE2 was obtained by amplifying M. tuberculosis genomic DNA with primers SigEPf and SigESr. The UreA derivative, pUreA, was constructed by cloning the PCR product amplified with primers UreAfw and UreArev into pPRB4. The annealing temperature was calculated independently for each primer

Table 1. Oligonucleotide primers used in this study

\begin{tabular}{|ll|}
\hline Primer & \multicolumn{1}{c|}{ Nucleotide sequence $\left(\mathbf{5}^{\prime}-\mathbf{3}^{\prime}\right)$} \\
\hline A & CGCGGATCCAACCGTATATAGTGCA \\
B & GCGGTACCCGGGATCTAAAATTATAAAA \\
BlaPf & CTCTGCAGTGCTCGGCGGACTCCGGGTGG \\
BlaPr & TTGGATCCATTGGACCCAGTGTAGCGGGT \\
BlaSr & TTGGATCCAGATTGGCTGCGTACAAGCCG \\
BlaSf & CTCTGCAGATGACCGGACTATCGCGACGC \\
SigEPf & AACTGCAGCGGCGACGTAATCTGGCTGGT \\
SigErev & CGAGGGGATCCATGGGAATTACCGT \\
SigESr & TTGGATCCGTCGTGATATTGAGATCCTCC \\
UreAfw & ACCCGCTCCGCTGAATGTCAC \\
UreArev & CCGCGGGCCCGACGCCGGCG \\
Nuc2 & AAACTGCAGCAACTTCAACTAAAAAATTAC \\
Rev & CAGGAAACAGCTATGACC \\
F & GTAAAACGACGGCCAGTGCCA \\
Nucr & AACCGTATCACCATCAATCGC \\
\hline
\end{tabular}

pair and 30 cycles were carried out. PCR products and plasmids were purified with the Qiagen PCR purification kit.

pNuc2 was constructed as follows. The blaF* promoter was inserted into pPV24 that had been digested with PstI and BamHI. The resulting plasmid was digested with BamHI and then with Mung Bean Nuclease (BioLabs) to remove the $5^{\prime}$ protruding ends, and then digested with KpnI. NucA was amplified with primers Nuc2 and Rev using pPRB4 as a template. The PCR product was digested with PstI, bluntended with T4 DNA polymerase and digested with KpnI. It was then cloned into the plasmid to give pNuc2. All the plasmids were first amplified in E. coli and sequenced.

Construction and screening of the fusion library. M. tuberculosis genomic DNA was partially digested with Sau3AI. Fragments of between 2 and $0.5 \mathrm{kbp}$ were excised from the gel and ligated into dephosphorylated pPRB4 which had been linearized with BamHI. Epicurian Coli XL2 BlueMRF' ultracompetent cells (Stratagene) were transformed with the ligation mixture and 24000 clones were obtained. Twelve clones were randomly picked and shown to be distinct by amplifying the inserts by PCR. The 24000 clones were pooled and plasmid DNA was extracted with a Qiagen Kit and used to transform M. smegmatis. We screened 32000 clones of transformed $M$. smegmatis for nuclease activity with the toluidine blue overlay assay. Inserts in the positive clones were amplified by PCR, using primers F and Nucr, and sequenced.

Detection of nuclease activity. To identify $\mathrm{Nuc}^{+}$clones on Petri dishes, a nuclease plate assay was used. Plates were overlaid with $12 \mathrm{ml}$ warm Toluidine Blue-DNA agar (TBDNA agar) (0.05 $\mathrm{M}$ Tris, $\mathrm{pH} 9,10 \mathrm{~g}$ agar $\mathrm{l}^{-1}, 10 \mathrm{~g} \mathrm{NaCl} \mathrm{l}{ }^{-1}$, $0 \cdot 1 \mathrm{mM} \mathrm{CaCl}, 0.03 \%$, w/v, salmon sperm DNA, $130 \mathrm{mg}$ toluidine blue $\mathrm{O} \mathrm{l}^{-1}$ ). Colonies which secreted active Nuc developed an easily detectable pink halo after 1 or $2 \mathrm{~h}$. A sterile net was placed over the colonies to ensure that they did not detach when the TB-DNA agar was poured. Alternatively, transformed and wild-type M. smegmatis were plated on methyl-green-containing plates (Downing et al., 1999). Colonies that secreted an active nuclease developed a yellow halo after $60 \mathrm{~h}$.

Zymograms were performed as follows. SDS gels were renaturated as described by Liebl et al. (1992), placed on a Petri dish containing TB-DNA agar and incubated at $37^{\circ} \mathrm{C}$. After $1-6 \mathrm{~h}$ pink bands indicated the presence of nuclease activity.

Cellular fractionation. Bacterial cells were grown in LB, supplemented with $0.05 \%$ Tween 80 , to an $\mathrm{OD}_{600}$ of $1.3-1 \cdot 8$ (mid-exponential phase) or 6.5 (stationary phase). The optical density was measured in $1 \mathrm{~cm}$ cuvettes in a Hitachi U-1100 spectrophotometer after a 1:10 dilution in LB. The bacteria were harvested by centrifuging $20 \mathrm{ml}$ culture at $4000 \mathrm{~g}$. The supernatant was filtered through a Millex-GV filter with $0 \cdot 22 \mu \mathrm{m}$ pores and precipitated with TCA ( $17 \%$ final volume). The pellet was washed, resuspended in $500 \mu \mathrm{l}$ PBS and the cells were mechanically broken with $0 \cdot 1 \mathrm{~mm}$ glass beads. The beads were removed by centrifugation and the suspension was precipitated with TCA. The precipitated samples were washed with cold acetone containing $1 \%$ triethanolamine. Finally, all the samples were resuspended in the appropriate volume of loading buffer and boiled for $5 \mathrm{~min}$.

Western blotting. SDS-PAGE and electroblotting were carried out by standard methods (Sambrook et al., 1989). The membrane was incubated in $5 \%$ milk PBST (PBS, $0.05 \%$ Tween 20) for $1 \mathrm{~h}$, washed briefly and incubated with antiKatG (diluted 1:1250 in 5\% milk PBST) or anti-Nuc (diluted 
1:1000 in 5\% milk PBST) antisera for $1 \mathrm{~h}$. After extensive washing in PBST the membrane was incubated for $1 \mathrm{~h}$ with a horseradish-peroxidase-conjugated anti-rabbit antibody (BioRad) (diluted 1:10000 in 5\% milk PBST). After extensive washing the immunodetection was performed by using an ECL kit (Amersham).

Preparation of anti-Nuc antibodies. New Zealand rabbits were immunized with $250 \mu \mathrm{g}$ commercial micrococcal nuclease (Sigma). Boosters $(200 \mu \mathrm{g})$ were given three times at 2week intervals. Blood was collected, incubated for $1 \mathrm{~h}$ at $37^{\circ} \mathrm{C}$ and overnight at $4{ }^{\circ} \mathrm{C}$. Sera were recuperated by centrifuging the blood at $3000 \mathrm{~g}$ for $20 \mathrm{~min}$ and tested by Western blotting.

Integration of 155nuc into $M$. smegmatis. pBla1* was constructed as follows. The PstI/KpnI fragment of pBla1 was blunt-ended and ligated into blunt-ended integrative $\mathrm{pNIP} 40 \mathrm{~b}$ that had been digested with $\mathrm{XbaI}$. The resulting plasmid was selected for the correct insert orientation and sequenced. Sequencing revealed that a guanine residue 54 bp upstream of the ATG site had been deleted. M. smegmatis was transformed with pBla1* and transformed clones were selected on hygromycin plates.

Transposon mutagenesis. M. smegmatis::pBla1* was transformed with pCG79 and transposon mutagenesis was carried out by the method of Guilhot et al. (1994), except that the colonies were plated out at $41^{\circ} \mathrm{C}$ to select clones in which transposition had occurred. After $3 \mathrm{~d}$ the colonies were tested for nuclease activity by the toluidine blue overlay assay. $\mathrm{Nuc}^{-}$ mutants were selected and analysed.

DNA sequencing. A model 373-B DNA analysis system (Applied Biosystems) was used to sequence the doublestranded plasmid DNA or PCR-generated fragments by the dideoxy chain-termination method.

Computer methods. Signal sequences were searched using the signal IP server at http://www.cbs.dtu.dk/services/SignalIP. Sequences were analysed by the BLAST algorithm at http:// www.sanger.ac.uk/Projects/M_tuberculosis/blast_server. shtml

\section{RESULTS}

\section{Screening of an $M$. tuberculosis-155Nuc fusion library in $M$. smegmatis}

In Staphylococcus aureus the nuclease protein is targeted for secretion by a 60 aa signal sequence. This signal sequence is removed during secretion to give rise to a
168 aa active protein, NucB. Subsequent proteolytic cleavage of its $19 \mathrm{~N}$-terminal amino acids, the propeptide, converts NucB into the 149 aa mature form, NucA.

An equivalent of the truncated nuclease used by Poquet et al. (1998), in which the signal sequence and the first 13 aa of the propeptide are deleted, was named $155 \mathrm{Nuc}$. The $155 n u c$ gene, deprived of its own promoter, was cloned into pPV24, a shuttle plasmid that replicates in both E. coli and mycobacteria (Triccas et al., 1999). The resulting vector was named pPRB4. M. smegmatis (pPRB4) was tested for nuclease activity by the toluidine blue overlay assay, a test which can detect just $5 \mathrm{ng}$ active nuclease $\mathrm{ml}^{-1}$ (Lachica et al., 1971) and which is non-toxic for the cells. Positive colonies can be readily detected after 1 or $2 \mathrm{~h}$ by their pink halo, which derives from the degradation of the DNA contained in the overlay. A $\mathrm{Nuc}^{-}$phenotype was observed for $M$. smegmatis(pPRB4), demonstrating the absence of a cryptic promoter and signal sequence in the vector and of endogenous nuclease. pPRB4 was subsequently used to construct an $M$. tuberculosis genomic library in fusion with the $5^{\prime}$ end of the 155 nuc gene. The library was amplified in E. coli and then used to transform $M$. smegmatis. Over 32000 M. smegmatis colonies were tested for nuclease activity by the toluidine blue overlay assay. Approximately $0 \cdot 15 \%$ of clones were positive after $1 \mathrm{~h}$. Some of the inserts contained in these clones were amplified and sequenced.

In $50 \%$ of the cases, (approx. 10 clones) $155 n u c$ was inframe with the beginning of a gene encoding a putative protein. However, a canonical signal sequence was predicted using the signal IP server in only $20 \%$ of the cases. These were Rv0347 and Rv2588c, both of which are of unknown function. Most other fusions were either with genes encoding cytosolic proteins (Rv0187, Rv1608c) or with a fragment that was too short to contain a signal sequence (Rv0270, Rv1837c) (Table 2). In the other $50 \%$ of the cases, the inserts were in intergenic or intragenic regions. In every case, however, a codon for methionine or valine, which may act as a start codon, was found in-frame with $155 \mathrm{nuc}$. This may be because $M$. tuberculosis genes are expressed in $M$. smegmatis and thus in a relatively heterologous context.

Table 2. Characteristics of the inserts of some $\mathrm{Nuc}^{+}$clones

\begin{tabular}{|llcc|}
\hline Gene & \multicolumn{1}{c}{ Predicted function } & Length of the insert (bp) & Amino acids in fusion \\
\hline Rv0347 & Unknown & 450 & 134 \\
Rv0270 & Acyl-CoA-synthase & 150 & 5 \\
Rv0187 & O-Methyltransferase & 250 & 23 \\
Rv0361 & Membrane protein* & 200 & 26 \\
Rv1837c & Malate synthase & 250 & 2 \\
Rv0360c & Unknown & 260 & 14 \\
Rv2588c & Unknown & 150 & 34 \\
Rv1608c & Bacterioferritin co-migratory & 150 & 13 \\
& protein & & \\
\hline
\end{tabular}

* The protein is predicted to have a transmembrane region between amino acids 132 and 156 . 


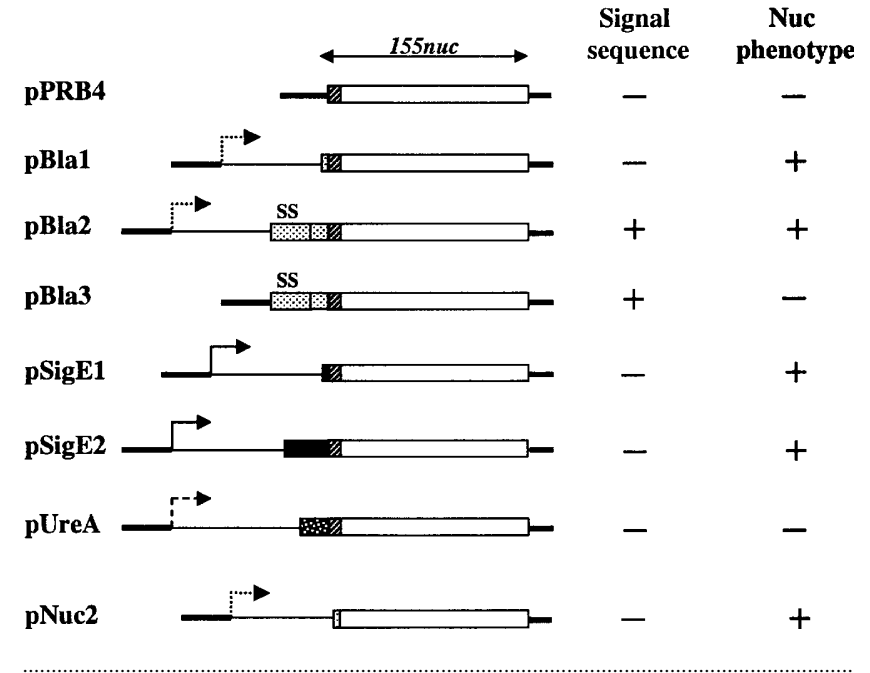

Fig. 1. Schematic representation of pPRB4 and its derivatives. 155 nuc is represented by an open box, the propeptide by a hatched box and the promoters are indicated by arrows. SS, signal sequence.

Some sequences in M. tuberculosis may be artefactually recognized as promoters, ribosome-binding sites and start codons by M. smegmatis. Alternatively, the genome may contain sequences that were not recognized for a particular annotation with the existing software. These results suggest that a promoter and some amino acids in-frame with the nuclease are sufficient to result in a Nuc ${ }^{+}$phenotype on the Petri dishes.

\section{Nuc is secreted by $M$. smegmatis independently of a signal sequence}

We subsequently attempted to determine whether the $\mathrm{Nuc}^{+}$phenotype seen on the Petri dishes corresponded to the secretion of the nuclease by M. smegmatis.

To determine whether a signal sequence was required for secretion a series of pPRB4 derivatives was constructed. In these derivatives $155 n u c$ was fused to (i) a promoter and signal sequence, (ii) a promoter and start codon, or (iii) a signal sequence alone. We aimed to verify whether the nuclease is secreted in the absence of a signal sequence as the data presented above suggested. We used the blaF* promoter and gene from Mycobacterium fortuitum, which encodes an exported $\beta$-lactamase with a 32 aa $\mathrm{N}$-terminal signal sequence (Timm et al., 1994). The blaF* gene was chosen because of its mycobacterial origin. In-frame fusions with $155 n u c$ were constructed by cloning the blaF* promoter and start codon (pBla1), the blaF* promoter and the 57 aa Nterminal sequence, which includes the signal sequence (pBla2), or the BlaF* N-terminal region without promoter (pBla3) into pPRB4 (Fig. 1). M. smegmatis was transformed with each of the constructs and was screened for Nuc activity by the toluidine blue overlay assay. As expected, M. smegmatis(pBla2) was $\mathrm{Nuc}^{+}$and M. smegmatis(pBla3) was $\mathrm{Nuc}^{-}$, thus confirming the absence of a cryptic promoter in pPRB4. Interestingly, a clear pink halo appeared in M. smegmatis(pBla1) at the same time and with the same intensity as in $M$. smegmatis(pBla2). This suggests that if an active promoter controls its expression, $155 \mathrm{Nuc}$ is efficiently secreted in M. smegmatis, even in the absence of a signal sequence.

To confirm that $155 \mathrm{Nuc}$ could be secreted by $M$. smegmatis in the absence of a signal sequence, other fusions were constructed with cytosolic proteins, such as SigE and UreA (Fig. 1). SigE is an M. tuberculosis sigma factor (Wu et al., 1997) and is thus a cytosolic protein lacking a secretion signal sequence. The promoter and the start codon, or the promoter and the region encoding the first 40 aa of $s i g E$, were fused in-frame with $155 \mathrm{nuc}$, resulting in pSigE1 and pSigE2, respectively. M. smegmatis was transformed with these vectors and the transformants were tested for nuclease activity. Both $M$. smegmatis(pSigE1) and M. smegmatis(pSigE2) were $\mathrm{Nuc}^{+}$, demonstrating that the expression of $155 \mathrm{nuc}$ was sufficient for $155 \mathrm{Nuc}$ secretion in M. smegmatis. UreA, the A subunit of the M. tuberculosis urease (Clemens et al., 1995; Reyrat et al., 1995), was the other cytosolic protein used. In-frame fusions of the promoter and the region encoding the first 26 a of ureA with $155 \mathrm{nuc}$ (pUreA) resulted in $\mathrm{Nuc}^{-}$transformants in M. smegmatis(pUreA). The difference in Nuc activity between M. smegmatis(pSigE2) and M. smegmatis(pUreA) is probably due to the different fragments that were fused to $155 n u c$ in the two vectors. It is probable that the $\mathrm{N}$ terminus of UreA prevents $155 \mathrm{Nuc}$ translocation or activity, whereas the $\mathrm{N}$ terminus of SigE does not.

To demonstrate that the $\mathrm{Nuc}^{+}$phenotype observed was due to protein secretion and not due to cell lysis we assayed the bacterial cell and supernatant fractions of mid-exponential-phase cultures for the presence of nuclease by Western blotting and for nuclease activity by zymogram analysis (Fig. 2). Cell lysis was monitored by Western blotting with an antibody against KatG, a catalase peroxidase (Zhang et al., 1992) exclusively cytoplasmic in M. smegmatis (Raynaud et al., 1998). This confirmed the previous observations and revealed that over $40 \%$ of the nuclease hybrid proteins was in the supernatant fraction, whereas KatG was exclusively found in the cell fraction. Furthermore, zymograms showed that all the hybrids had a distinct nuclease activity, demonstrating that the fused polypeptides did not prevent nuclease activity of $155 \mathrm{Nuc}$. The activity of the UreA-155Nuc fusion demonstrated that the absence of the halo was not due to a lack of activity, but that the hybrid protein was not secreted. One may argue that the presence of the nuclease in the supernatant could be due to the fact that it is a small protein $(15 \mathrm{kDa})$ and it can easily 'leak', contrary to KatG which is a protein of high molecular mass $(80 \mathrm{kDa})$ and is retained in the cells. The absence of UreA-Nuc $(17 \mathrm{kDa})$ in the supernatant demonstrates that the low molecular mass cannot explain the secretion per se.

To demonstrate formally that KatG was absent from the 


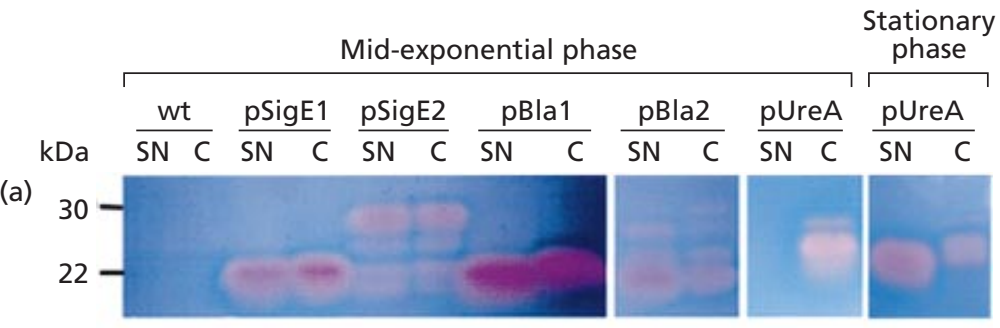

(b)

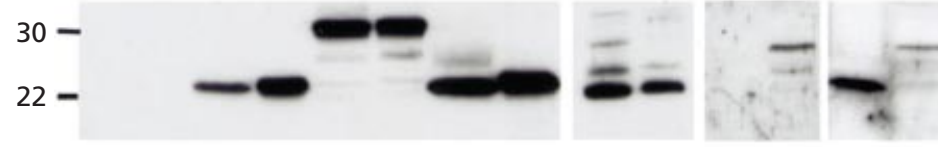

(c)

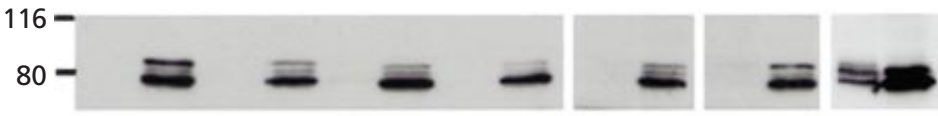

Fig. 2. (a) Zymogram, (b) Western blot with anti-Nuc serum and (c) Western blot with anti-KatG serum of supernatant (SN) and cell (C) fractions. Bacteria, equivalent to an $\mathrm{OD}_{600}$ of 1 , were loaded in each lane (ca 2 and $80 \mu \mathrm{g}$ in $\mathrm{SN}$ and in C fractions respectively, for mid-exponential-phase cultures). wt, $M$. smegmatis $\mathrm{mc}^{2}$ wild-type; pSigE1, M. smegmatis(pSigE1); pSigE2, M. smegmatis (pSigE2); pBla1, M. smegmatis(pBla1); pBla2, M. smegmatis(pBla2); pUreA, M. smegmatis (pUreA). extracellular medium due to an absence of lysis rather than the insolubility or instability of KatG in the culture medium, or the affinity of KatG for the cell wall, we carried out a Western blot with a stationary-phase culture. M. smegmatis(pUreA) was grown to an $\mathrm{OD}_{600}$ of 6.5 and both the supernatant and cellular fractions were analysed by Western blotting and zymogram. Under these conditions, KatG was also present in the supernatant fraction (Fig. 2). Thus, KatG is both soluble and stable in the culture medium and is therefore a good reporter of bacterial lysis in M. smegmatis. Under these conditions, UreA-155Nuc was also detected in the supernatant fraction. The nuclease detected in the supernatant fraction had a lower molecular mass than UreA-155Nuc, probably due to the proteolytic removal of the amino acids corresponding to UreA. Under these conditions the nuclease accumulated in the supernatant fraction, demonstrating that it is a highly stable protein.

M. smegmatis(pBla2) contained different bands, some of which may result from the cleavage of its signal sequence. The abnormal migration and the presence of degradation products that could represent the NucA form have been described (Poquet et al., 1998).

\section{Nuc secretion is due neither to overexpression nor to the propeptide}

Previous experiments used a derivative of the pAL5000 replicon, which is a replicative plasmid present at about three copies per bacterium (Ranes et al., 1990). To exclude the possibility that the secretion of the nuclease was due to its overexpression, we used pNIP40b, an integrative vector present in a single copy. The blaF* promoter and $155 \mathrm{nuc}$ of pBla1 were cloned into pNIP40b and the resulting pBla1* was integrated into M. smegmatis. M. smegmatis::pBla1* colonies remained $\mathrm{Nuc}^{+}$on Petri dishes, even though a longer incubation time was required. As in $M$. smegmatis (pBla1), fractionation showed that $155 \mathrm{Nuc}$ was in the

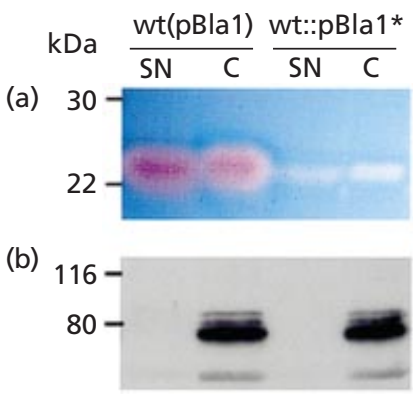

Fig. 3. (a) Zymogram and (b) Western blot with anti-KatG serum of supernatant (SN) and cell (C) fractions. $M$. smegmatis(pBla1), equivalent to an $\mathrm{OD}_{600}$ of 1 , and $M$. smegmatis::pBla $1 *$, equivalent to an $\mathrm{OD}_{600}$ of 2 , were loaded in the lanes. wt, M. smegmatis $\mathrm{mc}^{2}$ wild-type.

supernatant in the absence of cell lysis (Fig. 3). Although the Nuc level was greatly diminished in single-copy expression, $50 \%$ of nuclease activity was still found in the culture supernatant fraction. This demonstrates that $155 \mathrm{Nuc}$ secretion was not due to overexpression.

We also tested all the M. smegmatis recombinants with the methyl green plate assay (Downing et al., 1999). The bacteria which were positive in the toluidine blue overlay test were also positive on methyl green plates. Unlike the protein used as a reporter system by Downing et al. (1999), 155Nuc carries the last 6 aa of the propeptide. It has been demonstrated that in E. coli the propeptide dramatically enhances secretion because the fusion of a signal sequence with $\mathrm{NucB}$ is secreted much more efficiently than the fusion with NucA (Suciu \& Inouye, 1996). Thus, we hypothesized that part of this propeptide could be responsible for $155 \mathrm{Nuc}$ secretion in M. smegmatis. pNuc2, a replicative plasmid in which NucA (devoid of the propeptide) is directly controlled by the bla $F^{*}$ promoter, was constructed and tested in $M$. smegmatis (Fig. 1). M. smegmatis (pNuc2) colonies had 
Table 3. Characterization of the Nuc mutants in transposon mutant libraries

\begin{tabular}{|c|c|c|}
\hline Bank & Colonies tested & Mutant characterization \\
\hline 1 & 5000 & $1-10$ : absence of amplification of $155 n u c$ \\
\hline 2 & 12000 & $\begin{array}{l}\text { 11: absence of amplification of } 155 n u c \text { and } b l a F^{*} \text { promoter } \\
\text { 12: substitution } \operatorname{Leu}_{103} \rightarrow \operatorname{Ser}_{103}\end{array}$ \\
\hline 3 & 3000 & $\begin{array}{l}\text { 13: deletion of adenine }{ }_{153} \text { and creation of a stop codon } 6 \mathrm{bp} \\
\text { downstream }\end{array}$ \\
\hline 4 & 5000 & No Nuc${ }^{-}$mutants found \\
\hline
\end{tabular}

identical phenotypes to M. smegmatis(pBla1) in plate tests (data not shown). This indicated that $155 \mathrm{Nuc}$ secretion is not triggered by the $\mathrm{C}$ terminus of the propeptide in M. smegmatis.

\section{Search for alternative secretion pathways}

Our results clearly demonstrate that $155 \mathrm{Nuc}$ is secreted independently of overexpression, the presence of the propeptide and of the signal sequence. $155 \mathrm{Nuc}$ is thus probably secreted independently of the Sec translocating pathway. To characterize this alternative pathway we used M. smegmatis::pBla1* to create a library of Tn611 insertional mutants using the thermosensitive replicon pCG79 as a delivery system (Guilhot et al., 1994). The rationale was that we could distinguish $\mathrm{Nuc}^{-}$mutants on plate, mutants that could arise from mutations of the nuc gene itself or more interestingly in genes involved in 155 Nuc secretion. Such mutants would display cytosolic nuclease activity. Four independent libraries were constructed and 25000 clones were screened by a plate overlay test. Thirteen $\mathrm{Nuc}^{-}$mutants were found and analysed for the presence of 155 nuc by PCR with different pairs of primers and for nuclease activity by the zymogram assay. None of the mutants showed any nuclease activity in the zymogram assay when culture supernatants and cell fractions were tested (data not shown). This suggests that the nuclease gene was either not transcribed or not translated, or the protein was enzymically inactive. Indeed it was not possible to amplify either the promoter region or the promoter and the $155 n u c$ region in mutants $1-11$ (Table 3 ). This was probably due to deletions or to the insertion of the transposon in the $155 n u c$ gene or in the bla $F^{*}$ promoter. Two mutants (12 and 13) had point mutations in the $\mathrm{C}$ terminus: in mutant 12 a substitution had occurred in the second helix, responsible for $\mathrm{Ca}^{2+}$ binding (Hynes \& Fox, 1991), whereas in mutant 13 a deletion created a stop codon $6 \mathrm{bp}$ downstream, thus eliminating the Cterminal region of the protein (Table 3). No mutant with a Nuc ${ }^{-}$phenotype was found outside the nuc gene itself that could provide evidence for a new secretion pathway. Indeed, no mutant that retained a nuclease activity which was exclusively cytosolic could be identified, suggesting that either the nuclease is secreted by a nonspecific or redundant system, or that components of the secretion apparatus may be essential for bacterial survival.

\section{DISCUSSION}

Much effort was made to characterize the secreted products of M. tuberculosis. Gene fusions with reporter systems and computer methods have led to the characterization of some secreted products, but many of them remain uncharacterized. As for other Actinomycetes, $M$. tuberculosis secretes a vast number of proteins: more than 350 spots have been resolved by two-dimensional gel electrophoresis of $M$. tuberculosis culture filtrates (Rosenkrands et al., 2000). There are several examples of leaderless secretion in mycobacteria: for example, glutamine synthetase (Harth \& Horwitz, 1997), superoxide dismutase (Harth \& Horwitz, 1999) and Esat-6 (Sorensen et al., 1995), which are secreted in the absence of a signal sequence. Harth \& Horwitz (1999) have also shown that secretion is dependent upon both the information in the protein and the presence of special machinery present in both $M$. tuberculosis and $M$. smegmatis. However, to date the molecular mechanism responsible for this export remains to be characterized. Genome sequencing (Cole et al., 1998) has revealed the presence of the Sec and the TAT translocation pathways (Berks et al., 2000), and of many members of the ABC transporter family (Braibant et al., 2000) in M. tuberculosis. Genes homologous to those involved in type III and IV secretion have not been found. However, additional secretion pathways that allow signalsequence-independent secretion may exist in mycobacteria.

We used controlled conditions and a cytoplasmic marker to demonstrate that the staphylococcal nuclease is exported by $M$. smegmatis independently of a signal sequence. We showed that this protein is secreted by $M$. smegmatis whether it contains a signal sequence or not and even when fused to a short polypeptide. However, another fusion product was not secreted, probably because the polypeptide prevented the proper folding required for secretion or correct interactions with the secretion machinery.

Interestingly, a similar reporter system, also based on the staphylococcal nuclease, was used to characterize three exported products in M. tuberculosis (Downing et al., 1999). Our results clearly show that, on the contrary, the nuclease should not be used as a reporter system for the identification of exported products in mycobacteria because it is secreted independently of a signal sequence. 
In any case, we do not have any obvious explanation for such a discrepancy between the two results.

The nuclease protein sequence does not contain any classical signal sequences or long hydrophobic stretches that might explain its secretion. It is possible that the nuclease uses an endogenous alternative Sec pathway that could be an ABC transporter. For instance, Gey van Pittius et al. (2000) have suggested that Esat6, a small secreted antigen of M. tuberculosis which lacks a signal sequence (Sorensen et al., 1995), might use an ABC transporter for secretion. Also other species of Grampositive bacteria secrete small peptides without Secdependent signals via ABC transporters (Sahl \& Bierbaum, 1998). Porins and efflux pumps have also been described in mycobacteria (Trias et al., 1992), but, with a molecular mass of $\sim 15 \mathrm{kDa}$, it is unlikely that the nuclease is transported by such a system. As for the TAT pathway, the nuclease is devoid of signal peptide and consecutive arginines, both of which are required for efficient export and thus, the nuclease probably does not use this translocation pathway.

The fact that UreA-Nuc is not secreted demonstrates that secretion depends on both the sequence and the proper folding of the protein, as already suggested for other mycobacterial proteins (Harth \& Horwitz, 1997, 1999). The system which allows the secretion of this leaderless protein is present in M. smegmatis as for glutamine synthetase and superoxide dismutase (Harth \& Horwitz, 1997, 1999). Whether the secretion of all these leaderless proteins in M. smegmatis is mediated by the same translocation pathway or by different systems remains to be clarified.

The system that mediates the secretion of the nuclease could not be identified by transposition mutagenesis, despite the fact that more than 25000 clones, sufficient to cover the entire genome, were analysed. $\mathrm{Nuc}^{-}$ mutants resulted exclusively from mutations of the nuc gene itself, which strongly suggests that the genes encoding components of the alternative system may be essential. Alternatively, $155 \mathrm{Nuc}$ might be secreted through different systems that may be redundant and thus a single mutation cannot prevent secretion. Exported products play a key role in $M$. tuberculosis virulence and the identification of the pathways involved in their export is the next challenging step.

\section{NOTE ADDED IN PROOF}

Recently, extracellular localization of Mycobacterium tuberculosis glutamine synthetase and superoxide dismutase was shown to be due to high expression and extracellular stability (Tullius et al., 2001). Previous incorrect observations were due to a lack of a proper control to monitor autolysis.

\section{ACKNOWLEDGEMENTS}

C.R. is supported by a grant from the University of Padova (Italy). We are grateful to Philippe Langella for the gift of pVE3512, to François-Xavier Berthet for the early experi- ments with the nuclease, to Agnès Guigueno for the construction of pUreA and to Pilar Domenech Rubio for the gift of the anti-KatG serum. Tarek Msadek and Vladimir Pelicic are gratefully acknowledged for improving the manuscript. We also thank Antony Pugsley for encouraging discussions. J.M.R. is chargé de recherche at INSERM.

\section{REFERENCES}

Berks, B. C., Sergent, F. \& Palmer, T. (2000). The TAT protein export pathway. Mol Microbiol 35, 260-274.

Braibant, M., Gilot, P. \& Content, J. (2000). The ATP binding cassette (ABC) transport systems of Mycobacterium tuberculosis. FEMS Microbiol Rev 24, 449-467.

Braunstein, M., Griffin, T. J., Kriakov, J. I., Friedman, S. T., Grindley, N. D. F. \& Jacobs, W. R. (2000). Identification of genes encoding exported Mycobacterium tuberculosis proteins using a Tn522'phoA in vitro transposition system. J Bacteriol 182, 2732-2740.

Carroll, J. D., Wallace, R. C., Keane, J., Remond, H. G. \& Arbeit, R. D. (2000). Identification of Mycobacterium avium DNA sequences that encode exported proteins by using $p h o A$ gene fusions. Tuber Lung Dis 80, 117-130.

Chubb, A. J., Woodman, Z. L., da Silva Tatley, F., Jurgen Hoffmann, H., Scholle, R. \& Ehlers, M. (1998). Identification of Mycobacterium tuberculosis signal sequences that direct the export of a leaderless $\beta$-lactamase gene product in Escherichia coli. Microbiology 144, 1619-1629.

Clemens, D. L., Lee, B.-Y. \& Horwitz, M. A. (1995). Purification, characterization, and genetic analysis of Mycobacterium tuberculosis urease, a potentially critical determinant of hostpathogen interaction. J Bacteriol 177, 5644-5652.

Cole, S. T., Brosch, R., Parkhill, J. \& 39 other authors (1998). Deciphering the biology of Mycobacterium tuberculosis from the complete genome sequence. Nature 393, 537-544.

Downing, K. J., McAdam, R. A. \& Mizrahi, V. (1999). Staphylococcus aureus nuclease is a useful secretion reporter for mycobacteria. Gene 239, 293-299.

Gey van Pittius, N. C., Hide, W., Gamieldien, J., Brown, G. D. \& Beyers, A. D. (2000). Genomes 2000: International Conference on Microbial Model Genomes, abstract 1, p. 33.

Gomez, M., Johnson, S. \& Gennaro, M. L. (2000). Identification of secreted proteins of Mycobacterium tuberculosis by a bioinformatic approach. Infect Immun 68, 2323-2327.

Guilhot, C., Gicquel, B. \& Martin, C. (1992). Temperature-sensitive mutants of the Mycobacterium plasmid pAL5000. FEMS Microbiol Lett 98, 181-186.

Guilhot, C., Otal, I., Van Rompaey, I., Martin, C. \& Gicquel, B. (1994). Efficient transposition in mycobacteria: construction of Mycobacterium smegmatis insertional mutant libraries. $J$ Bacteriol 176, 535-539.

Harth, G. \& Horwitz, M. A. (1997). Expression and efficient export of enzymatically active Mycobacterium tuberculosis glutamine synthetase in Mycobacterium smegmatis and evidence that the information for the export is contained within the protein. $J$ Biol Chem 272, 22728-22735.

Harth, G. \& Horwitz, M. A. (1999). Export of recombinant Mycobacterium tuberculosis superoxide dismutase is dependent upon both information in the protein and mycobacterial export machinery. J Biol Chem 274, 4281-4292.

Hynes, T. R. \& Fox, R. O. (1991). The crystal structure of staphylococcal nuclease refined at $1.7 \AA$ resolution. Proteins Struct Funct Genet 10, 92-105. 
Lachica, R. V. F., Genigeorgis, C. \& Hoeprich, P. D. (1971). Metachromatic agar-diffusion methods for detecting staphylococcal nuclease activity. Appl Microbiol 21, 585-587.

Liebl, W., Sinskey, A. J. \& Schleifer, K.-H. (1992). Expression, secretion and processing of staphylococcal nuclease by Corynebacterium glutamicum. J Bacteriol 174, 1854-1861.

Lim, E. M., Rauzier, J., Timm, J., Torrea, G., Murray, A., Giquel, B. \& Portnoi, D. (1995). Identification of Mycobacterium tuberculosis DNA sequences encoding exported proteins by using $p h o A$ gene fusions. J Bacteriol 177, 59-65.

Poquet, I., Ehrlich, S. D. \& Gruss, A. (1998). An export-specific reporter designed for Gram-positive bacteria: application to Lactococcus lactis. J Bacteriol 180, 1904-1912.

Ranes, M. G., Rauzier, J., Lagranderie, M., Gheorghiu, M. \& Gicquel, B. (1990). Functional analysis of pAL5000, a plasmid from Mycobacterium fortuitum: construction of a 'mini' Mycobacterium-Escherichia coli shuttle vector. J Bacteriol 172, 27932797.

Ravn, P., Arnau, J., Madsen, S., Vrang, A. \& Israelsen, H. (2000). The development of TnNuc and its use for the isolation of novel secretion signals in Lactococcus lactis. Gene 242, 347-356.

Raynaud, C., Etienne, G., Peyron, P., Lanéelle, M. A. \& Daffé, M. (1998). Extracellular enzyme activities potentially involved in the pathogenicity of Mycobacterium tuberculosis. Microbiology 144, 577-587.

Reyrat, J. M., Berthet, F. X. \& Gicquel, B. (1995). The urease locus of Mycobacterium tuberculosis and its utilization for the demonstration of allelic exchange in Mycobacterium bovis bacillus Calmette-Guerin. Proc Natl Acad Sci U S A 92, 8768-8772.

Rosenkrands, I., Weldingh, K., Jacobsen, S., Veggerby Hansen, C., Florio, W., Gianetri, I. \& Andersen, P. (2000). Mapping and identification of Mycobacterium tuberculosis proteins by twodimensional gel electrophoresis, microsequencing and immunodetection. Electrophoresis 21, 935-948.

Sahl, H. G. \& Bierbaum, G. (1998). Lantibiotics: biosynthesis and biological activities of uniquely modified peptides from Grampositive bacteria. Annu Rev Microbiol 52, 41-79.

Sambrook, J., Fritsch, E. F. \& Maniatis, T. (1989). Molecular Cloning: a Laboratory Manual, 2nd edn. Cold Spring Harbor, NY: Cold Spring Harbor Laboratory.
Sorensen, A. L., Nagai, S., Houen, G., Andersen, P. \& Andersen, A. B. (1995). Purification and characterization of a low-molecularmass T-cell antigen secreted by M. tuberculosis. Infect Immun 63, $1710-1717$.

Suciu, D. \& Inouye, M. (1996). The 19-residue pro-peptide of staphylococcal nuclease has a profound secretion-enhancing ability in Escherichia coli. Mol Microbiol 21, 181-195.

Timm, J., Perilli, M. G., Duez, C. \& 9 other authors (1994). Transcription and expression analysis, using $l a c Z$ and $p h o A$ gene fusions, of Mycobacterium fortuitum $\beta$-lactamase genes cloned from a natural isolate and a high level $\beta$-lactamase producer. Mol Microbiol 12, 491-504.

Trias, J., Jarlier, V. \& Benz, R. (1992). Porins in cell wall of mycobacteria. Science 258, 1479-1481.

Triccas, J. A., Berthet, F.-X., Pelicic, V. \& Gicquel, B. (1999). Use of fluorescence induction and sucrose counterselection to identify Mycobacterium tuberculosis genes expressed within host cells. Microbiology 145, 2923-2930.

Tullius, M. V., Harth, G. \& Horwitz, M. A. (2001). High extracellular levels of Mycobacterium tuberculosis glutamine synthetase and superoxide dismutase in actively growing cultures are due to high expression and extracellular stability rather than to a protein-specific export mechanism. Infect Immun 69, 6348-6363.

Weldingh, K., Rosenkrands, I., Jacobsen, S., Birk Rasmussen, P., Elhay, M. J. \& Andersen, P. (1998). Two-dimensional electrophoresis for analysis of Mycobacterium tuberculosis culture filtrate and purification and characterization of six novel proteins. Infect Immun 66, 3492-3500.

Wiker, H. G., Wilson, M. A. \& Schoolnik, G. K. (2000). Extracytoplasmic proteins of Mycobacterium tuberculosis - mature secreted proteins often start with aspartic acid and proline. Microbiology 146, 1525-1533.

Wu, Q. L., Kong, D. \& Husson, R. N. (1997). A mycobacterial extracytoplasmic function sigma factor involved in survival following stress. J Bacteriol 179, 2922-2929.

Zhang, Y., Heym, B., Allen, B., Young, D. \& Cole, S. T. (1992). The catalase-peroxidase gene and isoniazid resistance of Mycobacterium tuberculosis. Nature 358, 591-593.

Received 30 July 2001; accepted 8 October 2001. 